\title{
Radiation therapy for nonmetastatic medically inoperable upper-tract urothelial carcinoma
}

\author{
Ming-Zhu Liu ${ }^{1} \wedge$, Xian-Shu Gao ${ }^{1}$, Shang-Bin Qin ${ }^{1}$, Xiao-Ying $\mathrm{Li}^{1}$, Ming-Wei Ma ${ }^{1}, \mathrm{Mu} \mathrm{Xie}^{1}$, Feng $\mathrm{Lyu}^{1}$, \\ Dian Wang ${ }^{2}$
}

${ }^{1}$ Department of Radiation Oncology, Peking University First Hospital, Peking University, Beijing, China; ${ }^{2}$ Department of Radiation Oncology, Rush University Medical Center, Chicago, IL, USA

Contributions: (I) Conception and design: XS Gao, D Wang; (II) Administrative support: XY Li, MW Ma; (III) Provision of study materials or patients: SB Qin, XY Li; (IV) Collection and assembly of data: MZ Liu; (V) Data analysis and interpretation: MZ Liu, M Xie, F Lyu; (VI) Manuscript writing: All authors; (VII) Final approval of manuscript: All authors.

Correspondence to: Xian-Shu Gao, MD. Department of Radiation Oncology, Peking University First Hospital, Peking University, Beijing, China. Email: doctorgaoxs@126.com; Dian Wang, MD. Department of Radiation Oncology, Rush University Medical Center, Chicago, IL, USA. Email: dian_wang@rush.edu.

Background: The standard management for upper urinary tract urothelial carcinoma (UTUC) is radical nephroureterectomy (RNU). However, some patients cannot undergo this procedure for several reasons, such as unresectable disease, old age, and multiple comorbidities. Our study explored the potential safety and effectiveness of radiotherapy as a curative treatment for UTUC patients unfit for surgery.

Methods: The data of patients treated with radiotherapy between December 2017 and November 2019 were retrospectively reviewed. For the literature review, computerized PubMed Medline, Index Medicus, and Web of Science databases and reference lists from the identified publications of interest were used. And "upper-tract urothelial carcinoma" and "radiotherapy" were used as key words in the search.

Results: We describe 8 patients with UTUC who were treated with radiotherapy. The median follow-up time was 13.5 months (range, 8.6-30.9 months). Local tumor control was achieved in all patients. However, distant metastases were observed in 2 patients with $\mathrm{T} 3-4 / \mathrm{N}+$ status. One patient had $\mathrm{T} 4$ status and the other had $\mathrm{N} 2+$ status. The patients died of tumor progression at 15.0 and 17.7 months. In addition, the other 6 patients who were still alive had relatively early-stage tumors without nodal involvement. Regarding acute toxicity, according to the CTCAE v5.0, mild side effects were noted, including grade 1 nausea and diarrhea. Four patients developed mild anemia, generally of grade $1-2$. One patient experienced grade 3 anemia, but it was manageable and improved with symptomatic support. In addition, no grade 4 acute or late toxicities were observed. No significant long-term impairment of renal function occurred.

Conclusions: For patients with nonmetastatic UTUC who are not suitable for surgery, radiotherapy is a safe treatment and can achieve good local tumor control.

Keywords: Upper urinary tract urothelial carcinoma (UTUC); radiation therapy; nonmetastatic; inoperable

Submitted Apr 08, 2021. Accepted for publication Jun 01, 2021.

doi: $10.21037 /$ tau-21-291

View this article at: https://dx.doi.org/10.21037/tau-21-291

^ ORCID: 0000-0003-2430-6517. 


\section{Introduction}

Upper urinary tract urothelial carcinoma (UTUC) is an uncommon disease and accounts for only $5-10 \%$ of urothelial carcinoma cases $(1,2)$. However, the incidence of UTUC has been increasing in recent years, and UTUC is generally most prevalent in China and the Balkan region (2). Consumption of aristolochic acid-containing Chinese herbs and Balkan endemic nephropathy are associated with an increased risk of urothelial carcinoma (3-5).

Surgery is the preferred approach for treating nonmetastatic UTUC, and radical nephroureterectomy (RNU) is the mainstay treatment for most patients (2). However, some patients may not be able to undergo surgery because of unresectable disease, old age, multiple comorbidities, and/or severely compromised performance status. UTUC has a peak incidence in individuals aged 70-90 years (6). Unfortunately, for patients unsuitable for surgery, the nonsurgical treatment strategy remains unclear due to a lack of research and effective clinical evidence. Nir Kleinmann reported that intracavitary administration of pyelocalyceal UGN-101, a mitomycincontaining reverse thermal gel, might offer a kidney-sparing treatment alternative (7) but mainly for low-grade upper tract urothelial carcinoma. Therefore, there is no standard treatment for patients with UTUC who are not candidates for surgery.

Radiotherapy has been used to treat patients with cancer for over 100 years. It is an effective tool in the armamentarium against many tumors, including bladder cancer and many other cancers. Radiotherapy is an alternative to cystectomy in patients with muscle-invasive bladder cancer (8-11). Particularly for elderly patients with invasive bladder cancer, radiotherapy can provide excellent results with a high complete response rate and good tolerability $(12,13)$. The main pathologic type of bladder cancer is urothelial carcinoma. Radiotherapy may offer a therapeutic alternative in such patients with UTUC who are unfit for surgery. With the development of radiotherapy technology, the application of volumetric modulated arc therapy (VMAT) can increase the dose in the tumor area to cure tumors while reducing the dose in the organs at risk (OARs), especially the intestines and spinal cord. Iizumi et al. found that radiotherapy may be a potential option for patients with UTUC, especially for those unsuitable for radical surgery (14). Consequently, radiotherapy may also be used as a noninvasive radical treatment to improve survival for such patients.

To date, in our institution, 8 patients who were unfit for surgery have been treated with VMAT. Our study reviews the cases of UTUC patients who were unfit for surgery and received radiotherapy and reviews the literature on this topic. We present the following article in accordance with the STROBE reporting checklist (available at https:// dx.doi.org/10.21037/tau-21-291).

\section{Methods}

\section{Patient selection}

From December 2017 to November 2019, a total of 8 patients with UTUC were treated with radiotherapy at the Peking University First Hospital and these cases were retrospectively analyzed. Computed tomography (CT) was performed on patients to confirm the location of the tumor and the absence of evidence of malignancy in the bladder and urethra. And 5 of 8 patients performed Cystoscopy or ${ }^{18} \mathrm{~F}$-fluorodeoxyglucose (18F-FDG) positron emission tomography (PET)/CT. Most patients refused to undergo invasive testing, such as ureteroscopic histologic biopsy. However, they underwent urine cytology testing, and 3 patients' urinary cytology results were positive for urothelial carcinoma. All patients underwent CT urography. Two experienced radiologists independently evaluated the scans to ensure that all medical images had minimal heterogeneity in the diagnosis, and disagreements were resolved by consensus (15).

Routine clinical staging included a complete medical history, physical examination, CT, PET/CT (if perfected). No patients exhibited evidence of distant metastatic disease. All patients were staged according to the 8th edition of the Union for International Cancer Control and American Joint Committee on Cancer (UICC/AJCC) staging system. The study was conducted in accordance with the Declaration of Helsinki (as revised in 2013). The study was approved by the institutional ethical committee of Peking University First Hospital (No.: 2016-1253) and individual consent for this retrospective analysis was waived.

\section{Treatments and follow-up procedures}

Before radiotherapy, the recruited patients underwent a CT scan (Big Bore 16-row helical CT with a 5-mm thick layer image reconstruction; Philips, Amsterdam, Netherlands) of the treatment area for planning purposes. A Synergy linear accelerator (6-MV photons, 60-pair multilobed grating; Elekta, Stockholm, Sweden) or a Trilogy linear accelerator (6-MV photons, 60-pair multilobed grating; Varian, Palo 
Table 1 Baseline clinical characteristics of patients

\begin{tabular}{|c|c|c|c|c|c|c|c|c|c|c|}
\hline No & $\begin{array}{c}\text { Age } \\
\text { (years) }\end{array}$ & Sex & KPS & Site & $\begin{array}{l}\text { Size } \\
(\mathrm{cm})\end{array}$ & TNM & Risk & RT fractionation & BED & Reason \\
\hline 1 & 84 & M & 70 & Renal pelvis & 2.7 & T3NOMO & High & SABR (24 Gy/3 fr) + CRT (52.8 Gy/22 fr) & 108.7 Gy & Refusal of surgery \\
\hline 2 & 60 & M & 70 & Renal pelvis & 3.2 & T1/2NOMO & High & SABR (30 Gy/5 fr) + CRT (48 Gy/20 fr) & 107.5 Gy & Medically inoperable \\
\hline 3 & 78 & $\mathrm{~F}$ & 80 & Renal pelvis & 2.6 & T1/2NOMO & High & SABR (24 Gy/3 fr) + CRT (52.8 Gy/22 fr) & 108.7 Gy & Medically inoperable \\
\hline 6 & 81 & M & 90 & Ureter & 3.3 & T3N2M0 & High & CRT (62.5 Gy/25 fr) & 78.1 Gy & Refusal of surgery \\
\hline 7 & 94 & M & 80 & Ureter & 5.1 & T1/2NOMO & High & CRT (60 Gy/25 fr) & 74.4 Gy & Refusal of surgery \\
\hline 8 & 84 & $M$ & 90 & Ureter & 2.4 & T2/3NOMO & High & CRT (67.5 Gy/25 fr) & 85.7 Gy & Refusal of surgery \\
\hline
\end{tabular}

$\mathrm{RT}$, radiotherapy; SABR, stereotactic ablative boost radiotherapy; BED, biologically equivalent dose; CRT, conventionally fractionated radiotherapy

Alto, CA) was used. Patients were immobilized with the thermoplastic film. All patients underwent image-guided VMAT (IG-VMAT) and daily cone-beam computerized tomography (CBCT) to ensure accuracy of the position and reduce errors.

\section{Radiotherapy technique}

The gross tumor volume (GTV), comprising the known extent of disease (primary and nodal) on imaging (CT/ PET-CT). The clinical target volume (CTV) includes regions of presumed microscopic extent or dissemination. The planning target volume (PTV) consisted of the CTV with a $5 \mathrm{~mm}$ margin expansion. There are two strategies for radiotherapy: partial stereotactic ablative boost radiotherapy (p-SABR) and conventionally fractionated radiotherapy (CRT). When the tumor was located in the renal pelvis and upper ureter, most patients were treated with p-SABR regimen because it's farther from the intestine. However, when the tumor was located in the middle and lower ureter, the CRT protocol was chosen and the field of CTV extended, including the adjacent ureteral passage region and the lymphatic drainage region.

Half of the 8 patients underwent the p-SABR regimen, which consisted of two phases. The focus of the p-SABR regimen is sequential dose administration, with SABR as the first stage of radiotherapy followed by the second stage of CRT. The SABR component delivered a GTV dose of 24-30 Gy in 3-5 fractions, and the subsequent CRT component delivered a dose of 40-52.8 Gy in 20-22 fractions. The total GTV dose was approximately
76.8-78 Gy. When assuming an $\alpha / \beta$ ratio of 10 , the biological equivalent dose $\left(\mathrm{BED}_{10}\right)$ of the tumor was 107.5108.7 Gy. The PTV dose was approximately 50-60 Gy in 25 fractions. The other 4 patients underwent the CRT regimen, which included delivery of a prescribed GTV dose ranging from 60 to $67.5 \mathrm{~Gy}$ and a PTV dose of 45-50 Gy in 25 fractions. The mean $\mathrm{BED}_{10}$ of the tumor was 78.1 Gy (74.4-85.7 Gy) (Table 1). During the SABR component, the dose in OARs decreased to approximately $3 \mathrm{~Gy} / \mathrm{f}$. The total dose limit of OARs was within the safe range specified in the Radiation Therapy Oncology Group (RTOG) standard.

\section{Follow-up procedures}

All patients were followed up at 3-6 months intervals after VMAT or until death, with intervals extending to 6-12 months after 2 years. Physical examination, CT and blood routine and renal function examination were needed. Toxicity was graded by the patient's treatment team according to the National Cancer Institute Common Toxicity Criteria for Adverse Events (CTCAE), version 5.0.

\section{Literature review and statistical analysis}

An extensive review of the literature on UTUC patients unfit for surgery who received radiotherapy was performed using the PubMed Medline, Index Medicus, and Web of Science databases and the reference lists from the identified publications of interest (review articles, case reports). "Upper-tract urothelial carcinoma" and "radiotherapy" 
Table 2 Treatment outcomes of patients

\begin{tabular}{|c|c|c|c|c|c|c|}
\hline No & Local recurrence & Distant metastasis & Metastasis site & $\begin{array}{l}\text { Time to metastasis } \\
\text { (months) }\end{array}$ & Death & Survival (months) \\
\hline 1 & No & No & - & - & No & 8.6 \\
\hline 3 & No & No & - & - & No & 11.0 \\
\hline 6 & No & Yes & Liver & 11.0 & Yes & 17.7 \\
\hline 7 & No & No & - & - & No & 12.0 \\
\hline 8 & No & No & - & - & No & 9.4 \\
\hline
\end{tabular}
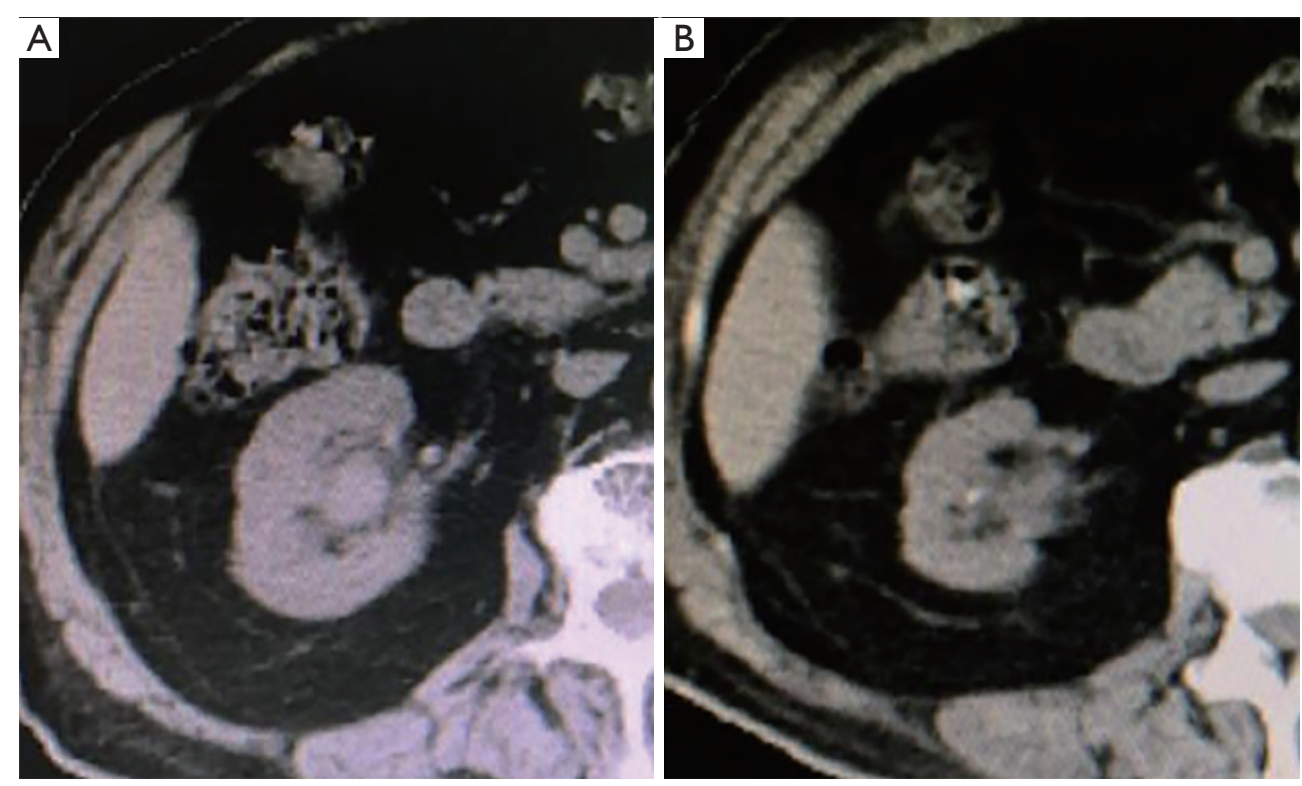

Figure 1 CT images after radical radiotherapy (case 1). The right renal pelvis tumor 1 week before and 3 months after RT. RT, radiotherapy.

were used as key words in the search. The clinical baseline data of the patients, mainly including sex, age, tumor site, clinical stage, radiation fractionation and field, and eventual multiplicity, were recorded and analyzed when available.

\section{Results}

The patient characteristics and treatments are summarized in Table 1 . The primary tumor sites included the ureter $(n=5)$ and renal pelvis $(n=3)$. The average age was 80.75 years (range, 60 to 94 years). All patients were treated with radiotherapy alone, without any systemic therapy. The median follow-up time was 13.5 months (range,
8.6-30.9 months). The median $\mathrm{BED}_{10}$ was $96.6 \mathrm{~Gy}$.

Local tumors were well controlled in all patients. Distant visceral metastasis occurred at 13.5 and 11.0 months in 2 patients. One patient had T4 status and the other had N2+ status. The 2 patients both died of tumor progression at 15.0 and 17.7 months. The other 6 patients had no disease progression and no recurrence or distant metastasis. At the last follow-up, the remaining 6 patients were still alive without disease progression (Table 2). After radiotherapy, local tumors can be well controlled, as shown in Figure 1. Ureteral stricture due to tumor mass could also be relieved under tumor control.

During the follow-up, in terms of acute toxicity, mild 
Table 3 Summary of treatment morbidities

\begin{tabular}{|c|c|c|c|}
\hline No & \multicolumn{2}{|c|}{ Acute } & Late [grade] \\
\hline 1 & Anemia [3] & None [0] & Anemia [1] \\
\hline 2 & None & Nausea [1] & None \\
\hline 3 & Anemia [2] & Diarrhea [1] & None \\
\hline 5 & None & Diarrhea [1], urinary frequency [1] & None \\
\hline 6 & Anemia [1] & None & None \\
\hline 7 & Anemia [1] & None & None \\
\hline 8 & None & None & None \\
\hline
\end{tabular}

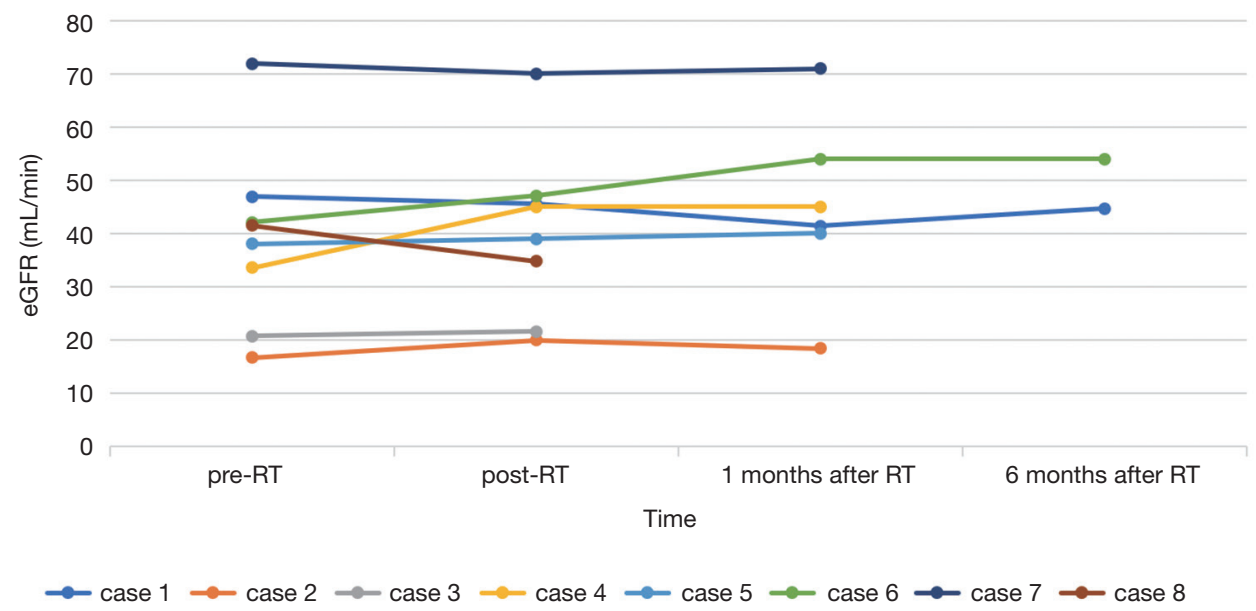

Figure 2 The eGFR before and after radiation for all patients with UTUC. eGFR, estimated glomerular filtration rate; RT, radiotherapy; UTUC, upper urinary tract urothelial carcinoma.

side effects were noted, including grade 1 nausea and diarrhea. Four patients developed mild anemia, generally of grade $1-2$. One patient experienced grade 3 anemia, but it was manageable and improved with symptomatic support. In addition, no grade 4 toxicities were observed. With respect to late toxicities, we observed no serious radiationassociated injuries (Table 3). There was no significant longterm impairment of renal function and no ureterostenosis occurred (Figure 2).

\section{Literature review}

According to the literature, 9 patients with UTUC who underwent radiotherapy and did not receive surgery were described in 3 publications $(14,16,17)$ (Table 4). In terms of the radiotherapy strategies used, two of the studies used photons, and one used proton.

\section{Discussion}

A new noninvasive treatment model is necessary for patients with UTUC who are medically inoperable. Radiotherapy plays a very important role in the treatment of urothelium carcinoma. Based on the above literature, radiotherapy can serve as a safe and effective noninvasive treatment for patients with nonmetastatic UTUC who cannot tolerate or refuse surgery.

Additionally, UTUC that invades the muscle wall usually 
Table 4 Radiotherapy for nonmetastatic, medically inoperable, UTUC: review of published cases

\begin{tabular}{|c|c|c|c|c|c|c|c|c|c|}
\hline $\begin{array}{l}\text { Case No } \\
\text { and } \\
\text { reference }\end{array}$ & $\begin{array}{c}\text { Age } \\
\text { (years) }\end{array}$ & Sex & Location & $\begin{array}{l}\text { Size } \\
(\mathrm{cm})\end{array}$ & TNM & Risk & $\mathrm{RT}$ fractionation & Chemotherapy & $\begin{array}{l}\text { Time to } \\
\text { progression } \\
\text { (months) }\end{array}$ \\
\hline $\begin{array}{l}\text { Maehata } \\
\text { et al. (17) }\end{array}$ & 87 & $M$ & Ureter & - & T2NOMO & - & $50 \mathrm{~Gy} / 10 \mathrm{fr}$ & No & 12 \\
\hline $\begin{array}{l}\text { Evans } \\
\text { et al. (16) }\end{array}$ & 95 & M & Renal pelvis & 4.5 & T2NOMO & High & $50 \mathrm{~Gy} / 4 \mathrm{fr}$ & No & - \\
\hline \multirow{3}{*}{$\begin{array}{l}\text { lizumi } \\
\text { et al. (14) }\end{array}$} & 72 & M & Renal pelvis & 3 & T1/2NOMO & High & $72.6 \mathrm{~Gy} / 22 \mathrm{fr}$ & No & - \\
\hline & 85 & - & Renal pelvis & 2 & T1/2NOMO & High & $72.6 \mathrm{~Gy} / 22 \mathrm{fr}$ & No & 36 \\
\hline & 64 & - & Renal pelvis & 4 & T4N2M1 & High & $66 \mathrm{~Gy} / 33 \mathrm{fr}$ & Yes & 28 \\
\hline \multirow{6}{*}{$\begin{array}{l}\text { Present } \\
\text { series }\end{array}$} & 84 & $M$ & Renal pelvis & 2.7 & T3NOMO & High & SABR (24 Gy/3 fr) + CRT (52.8 Gy/22 fr) & No & - \\
\hline & 60 & M & Renal pelvis & 3.2 & T1/2NOMO & High & SABR (30 Gy/5 fr) + CRT (48 Gy/20 fr) & No & - \\
\hline & 78 & $\mathrm{~F}$ & Renal pelvis & 2.6 & T1/2NOMO & High & SABR (24 Gy/3 fr) + CRT (52.8 Gy/22 fr) & No & - \\
\hline & 89 & $\mathrm{~F}$ & Ureter & 8.6 & T4NOMO & High & SABR (30 Gy/5 fr) + CRT (48 Gy/20 fr) & No & 13.5 \\
\hline & 76 & M & Ureter & 1.1 & T1/2NOMO & Low & CRT (62.5 Gy/25 fr) & No & - \\
\hline & 81 & M & Ureter & 3.3 & T3N2M0 & High & CRT (62.5 Gy/25 fr) & No & 11.0 \\
\hline
\end{tabular}

RT, radiotherapy; SABR, stereotactic ablative boost radiotherapy; CRT, conventionally fractionated radiotherapy; UTUC, upper urinary tract urothelial carcinoma.

has a poor prognosis. Several retrospective reviews have reported that the locoregional failure rate varies from $6.2 \%$ to $46.3 \%$ in UTUC patients who undergo RNU (18-20). The 5 -year cancer-specific survival (CSS) is $<50 \%$ for pT2/ pT3 and $<10 \%$ for pT4 UTUC (21-23). In our study, local tumors were well controlled in all patients. Although our follow-up time was not sufficiently long, local tumor control was still effective compared with that in previously reported studies. According to our retrospective analysis of the previous literature and a summary of previously reported cases, similar supporting conclusions can be drawn. The results of the analysis show that radiotherapy is beneficial for local tumor control in patients with nonmetastatic UTUC.

In terms of the radiation dose and fraction, the median
$\mathrm{BED}_{10}$ in this study was similar to that in previous studies (96.6 vs. $96 \mathrm{~Gy}$ ), most of which used limited field exposure, suggesting that the radiotherapy dose used in this study can achieve good local control.

In the current study, no local recurrence was observed until the last follow-up in the 8 patients, while the main measure for failure was distant metastasis. Previous studies have reported that systemic recurrences are frequent and lethal in advanced ( $\geq \mathrm{pT} 3$ ) UTUC stages (24). The 5 -year CSS rate of patients with early-stage $(\leq \mathrm{pT} 2 \mathrm{~N} 0)$ UTUC treated with RNU is fair (74.7\%); in contrast, the rates are decreased in patients with advanced disease (pT3, 54.0\% and pT4, 12.2\%) or nodal involvement (35.5\%) (25). Specific poor prognosis factors mainly include older age, poor 
performance status, tobacco consumption, hydronephrosis, high tumor stage and grade, and lymphatic metastasis (2). In our study, most of the enrolled patients were older, with an average age older than 80 years, and these patients had many poor prognostic factors. The two patients who died of tumor progression in this study also had advanced age and a high T/ $\mathrm{N}+$ stage. Local tumor control was achieved in all patients. Compared to the results of previous studies, these results indicate that radiotherapy can benefit local tumor control, but distant metastasis is the main reason for failure.

Regarding safety, two individual studies published in the last few years were case reports of the use of stereotactic body radiotherapy (SBRT) for the treatment of UTUC. Maehata and Evans both reported their experience using SBRT in elderly patients with medically inoperable UTUC, and no acute or late adverse events were observed in any of the three cases $(16,17)$. In the present study, our cases supported the clinical experience of Maehata and Evans. During treatment and follow-up, the plans were completed on schedule, and no severe acute or late complications were observed, even though 3 of the 8 patients were treated using extended radiotherapy fields. More specifically, no acute or late toxicities above grade 3 were observed, and although anemia and gastrointestinal discomfort were common, the cases were generally mild, with no significant impact on quality of life. Regarding renal function, there was no obvious impairment in the patients who could be observed. On the one hand, the reason may be that the tumor in most cases was located in the ureter and that the radiation dose to the kidney was low. However, longer follow-up periods are needed. In addition, no stricture was found during radiotherapy in our study or in previous reports. Some possible reasons are that the doses given in our current study are safe, with a very low probability of inducing stenosis, or that our follow-up time was not sufficiently long. On the other hand, in the case of UTUC, the small intestine is close to the ureter. Previously, it was very difficult for conventional radiotherapy to achieve a radical dose in the tumor area due to the dose limitation of normal tissues such as the small intestine. However, recent advances in radiotherapy technology have provided strategies that enable sparing of OARs from high doses of treatment, and the application of daily image guidance technology can also help reduce errors. Overall, our data have provided evidence that radiotherapy may be a safe radical treatment for patients with UTUC who are unfit for surgery.

Given the recent POUT results (26), adjuvant platinumbased chemotherapy should be considered as a new standard of care after nephroureterectomy for patients with UTUC. Therefore, the potential role of chemotherapy in medically inoperable UTUC may be worth studying in the future. Immunotherapy targeting the PD-1/PD-L 1 pathway has had good effects in treating urothelial tumors (27). It has been reported that radiotherapy has the potential to synergize with immunotherapy to improve oncological outcomes in patients with urothelial muscle-invasive bladder cancer (28). PD-L1 expression on tumor cells independently predicted shorter CSS in UTUC (29). Thus, the application of radiotherapy combined with immunization in UTUC may be a direction worthy of future exploration.

There were several limitations of this case report. First, 4D breath-gating techniques may be needed because the movement of the kidneys may vary with the degree of breathing motion. Second, the staging and diagnosis of UTUC were based on clinical findings and not pathological findings. Third, this report was a retrospective study. The durations of observation in this study were short, and the number of cases was small. Further studies including a larger number of patients are also needed. A longer observation period may be needed to more accurately estimate late adverse events.

\section{Conclusions}

For patients with nonmetastatic UTUC who are not suitable for surgery, radiotherapy is a safe treatment and can achieve good local tumor control. Future research should be conducted to confirm these results.

\section{Acknowledgments}

Funding: None.

\section{Footnote}

Reporting Checklist: The authors have completed the STROBE reporting checklist. Available at https://dx.doi. org/10.21037/tau-21-291

Data Sharing Statement: Available at https://dx.doi. org/10.21037/tau-21-291

Peer Review File: Available at https://dx.doi.org/10.21037/ tau-21-291

Conflicts of Interest: All authors have completed the ICMJE 
uniform disclosure form (available at https://dx.doi. org/10.21037/tau-21-291). The authors have no conflicts of interest to declare.

Ethical Statement: The authors are accountable for all aspects of the work in ensuring that questions related to the accuracy or integrity of any part of the work are appropriately investigated and resolved. The study was conducted in accordance with the Declaration of Helsinki (as revised in 2013). The study was approved by the institutional ethical committee of Peking University First Hospital (No.: 2016-1253) and individual consent for this retrospective analysis was waived.

Open Access Statement: This is an Open Access article distributed in accordance with the Creative Commons Attribution-NonCommercial-NoDerivs 4.0 International License (CC BY-NC-ND 4.0), which permits the noncommercial replication and distribution of the article with the strict proviso that no changes or edits are made and the original work is properly cited (including links to both the formal publication through the relevant DOI and the license). See: https://creativecommons.org/licenses/by-nc-nd/4.0/.

\section{References}

1. Siegel RL, Miller KD, Jemal A. Cancer statistics, 2019. CA Cancer J Clin 2019;69:7-34.

2. Rouprêt M, Babjuk M, Burger M, et al. European Association of Urology Guidelines on Upper Urinary Tract Urothelial Carcinoma: 2020 Update. Eur Urol 2021;79:62-79.

3. Wu F, Wang T. Risk assessment of upper tract urothelial carcinoma related to aristolochic acid. Cancer Epidemiol Biomarkers Prev 2013;22:812-20.

4. Lai MN, Wang SM, Chen PC, et al. Population-based case-control study of Chinese herbal products containing aristolochic acid and urinary tract cancer risk. J Natl Cancer Inst 2010;102:179-86.

5. Jelaković B, Karanović S, Vuković-Lela I, et al. Aristolactam-DNA adducts are a biomarker of environmental exposure to aristolochic acid. Kidney Int 2012;81:559-67.

6. Shariat SF, Favaretto RL, Gupta A, et al. Gender differences in radical nephroureterectomy for upper tract urothelial carcinoma. World J Urol 2011;29:481-6.

7. Kleinmann N, Matin SF, Pierorazio PM, et al. Primary chemoablation of low-grade upper tract urothelial carcinoma using UGN-101, a mitomycin-containing reverse thermal gel (OLYMPUS): an open-label, singlearm, phase 3 trial. Lancet Oncol 2020;21:776-85.

8. Coen JJ, Zhang P, Saylor PJ, et al. Bladder Preservation With Twice-a-Day Radiation Plus Fluorouracil/ Cisplatin or Once Daily Radiation Plus Gemcitabine for Muscle-Invasive Bladder Cancer: NRG/RTOG 0712-A Randomized Phase II Trial. J Clin Oncol 2019;37:44-51.

9. Ghate K, Brennan K, Karim S, et al. Concurrent chemoradiotherapy for bladder cancer: Practice patterns and outcomes in the general population. Radiother Oncol 2018;127:136-42.

10. James ND, Hussain SA, Hall E, et al. Radiotherapy with or without chemotherapy in muscle-invasive bladder cancer. N Engl J Med 2012;366:1477-88.

11. Rödel C, Grabenbauer GG, Kühn R, et al. Combinedmodality treatment and selective organ preservation in invasive bladder cancer: long-term results. J Clin Oncol 2002;20:3061-71.

12. Hammer L, Laufer M, Dotan Z, et al. Accelerated Hypofractionated Radiation Therapy for Elderly Frail Bladder Cancer Patients Unfit for Surgery or Chemotherapy. Am J Clin Oncol 2019;42:179-83.

13. Canyilmaz E, Yoney A, Serdar L, et al. Long-term results of a concomitant boost radiotherapy technique for elderly patients with muscle-invasive bladder cancer. J Geriatr Oncol 2015;6:316-23.

14. Iizumi T, Ishikawa $\mathrm{H}$, Sekino $\mathrm{Y}$, et al. Proton beam therapy for renal pelvis and ureter cancer: A report of 5 cases and a literature review. Mol Clin Oncol 2019;11:24-30.

15. Takeuchi M, Konrad AJ, Kawashima A, et al. CT Urography for Diagnosis of Upper Urinary Tract Urothelial Carcinoma: Are Both Nephrographic and Excretory Phases Necessary? AJR Am J Roentgenol 2015;205:W320-7.

16. Evans JD, Hansen CC, Tollefson MK, et al. Stereotactic body radiation therapy for medically inoperable, clinically localized, urothelial carcinoma of the renal pelvis: A case report. Adv Radiat Oncol 2018;3:57-61.

17. Maehata Y, Kuriyama K, Aoki S, et al. Stereotactic Body Radiotherapy for Localized Ureter Transitional Cell Carcinoma: Three Case Reports. Case Rep Urol 2015;2015:519897.

18. Li CC, Chang TH, Wu WJ, et al. Significant predictive factors for prognosis of primary upper urinary tract cancer after radical nephroureterectomy in Taiwanese patients. Eur Urol 2008;54:1127-34.

19. Wu CF, Pang ST, Chen CS, et al. The impact factors 
on prognosis of patients with p'T3 upper urinary tract transitional cell carcinoma. J Urol 2007;178:446-50, dicussion 450 .

20. Ozsahin M, Zouhair A, Villà S, et al. Prognostic factors in urothelial renal pelvis and ureter tumours: a multicentre Rare Cancer Network study. Eur J Cancer 1999;35:738-43.

21. Rouprêt M, Hupertan V, Seisen T, et al. Prediction of cancer specific survival after radical nephroureterectomy for upper tract urothelial carcinoma: development of an optimized postoperative nomogram using decision curve analysis. J Urol 2013;189:1662-9.

22. Lughezzani G, Burger M, Margulis V, et al. Prognostic factors in upper urinary tract urothelial carcinomas: a comprehensive review of the current literature. Eur Urol 2012;62:100-14.

23. Lughezzani G, Jeldres C, Isbarn H, et al. Nephroureterectomy and segmental ureterectomy in the treatment of invasive upper tract urothelial carcinoma: a population-based study of 2299 patients. Eur J Cancer 2009;45:3291-7.

24. Kim M, Kim JK, Lee J, et al. Adjuvant Treatments for Advanced Stage, Non-metastatic Upper Tract Urothelial

Cite this article as: Liu MZ, Gao XS, Qin SB, Li XY, Ma MW, Xie M, Lyu F, Wang D. Radiation therapy for nonmetastatic medically inoperable upper-tract urothelial carcinoma. Transl Androl Urol 2021;10(7):2929-2937. doi: 10.21037/tau-21-291
Carcinoma: A Multicenter Study. Int J Radiat Oncol Biol Phys 2019;104:819-27.

25. Margulis V, Shariat SF, Matin SF, et al. Outcomes of radical nephroureterectomy: a series from the Upper Tract Urothelial Carcinoma Collaboration. Cancer 2009;115:1224-33.

26. Birtle A, Johnson M, Chester J, et al. Adjuvant chemotherapy in upper tract urothelial carcinoma (the POUT trial): a phase 3, open-label, randomised controlled trial. Lancet 2020;395:1268-77.

27. Powles T, Eder JP, Fine GD, et al. MPDL3280A (antiPD-L1) treatment leads to clinical activity in metastatic bladder cancer. Nature 2014;515:558-62.

28. Daro-Faye M, Kassouf W, Souhami L, et al. Combined radiotherapy and immunotherapy in urothelial bladder cancer: harnessing the full potential of the anti-tumor immune response. World J Urol 2021;39:1331-43.

29. Zhang B, Yu W, Feng X, et al. Prognostic significance of PD-L1 expression on tumor cells and tumor-infiltrating mononuclear cells in upper tract urothelial carcinoma. Med Oncol 2017;34:94. 